This document is the accepted manuscript version of the following article:

Schuwirth, N., Kattwinkel, M., \& Stamm, C. (2015). How stressor specific are trait-based ecological indices for ecosystem management? Science of the Total Environment, 505, 565-572.

https://doi.org/10.1016/j.scitotenv.2014.10.029

\title{
How stressor specific are trait-based ecological indices for ecosystem management?
}

Nele Schuwirth", Mira Kattwinkel, Christian Stamm

Eawag: Swiss Federal Institute of Aquatic Science and Technology, 8600 Dübendorf,

Switzerland, nele.schuwirth@eawag.ch, mira.kattwinkel@eawag.ch,christian.stamm@eawag.ch

5 *corresponding author: nele.schuwirth@eawag.ch, phone: +41 (0)58 76555 28, Eawag, Überlandstrasse 133, P.O. Box 611, 8600 Dübendorf, Switzerland

Highlights:

- trait-based indices can help river managers to identify relevant stressors

- we test how stressor specific such indices are by analyzing the sensitivity of taxa

10 - we quantify inherent correlations between indices with Monte-Carlo simulations

- we demonstrate how to clearly distinguish these from correlations between stressors

- inherent correlation of indices increases with decreasing biodiversity 


\section{Abstract}

15 Using macroinvertebrates as ecological indicators for different stressors has a long tradition. However, when applied to field data, one often observes correlations between different macroinvertebrate indices that can be attributed to both correlations of stressors and inherent correlations due to the sensitivity of taxa to different stressors. Ignoring the source of any given correlation leads to ambiguous conclusions about the impact of different stressors.

20 Here, we demonstrate how to distinguish the causes of correlation by means of Monte Carlo simulations. We assessed to which degree trait-based indices are stressor-specific and whether this depends on the pool of taxa and its taxonomic resolution. Therefore, we (1) analysed the frequencies of "sensitive" and "insensitive" taxa for pairwise combinations of different indices, (2) analysed the inherent correlation of indices with random samples from different taxon pools 25 derived from field samples and from a complete species list of a whole ecoregion, (3) compared this inherent correlation with the actual correlation of the field samples. We exemplified this approach by analysing two existing indices $\left(\mathrm{SPEAR}_{\text {pesticides, }}\right.$ Saprobic Index) and new indices for temperature, flow and $\mathrm{pH}$ stress. We used these new indices to illustrate our approach while indepth testing of their applicability was not the focus of our study.

30 We found strong correlations between several indices in our study area at the Swiss Plateau. The probability that this correlation is only due to inherent correlation in the taxa sensitivities was low (maximum 0.35). The problem of inherent correlation between indices is more severe for the smaller taxon pool with lower taxonomic resolution.

Correlation in the sensitivity of different taxa to different stressors leads to an inherent

35 correlation in trait-based indices, which weakens their explanatory power. Our results highlight the importance of correlation analyses when using trait-based indices to guide ecosystemmanagement, especially in regions with reduced biodiversity.

Keywords: River management, bioassessment, macroinvertebrates, multiple stressors, traitbased indices, independence, Monte Carlo simulations

\section{Introduction}

In many regions of the world, freshwater ecosystems are heavily impacted by humans and suffer from various sources of impairment (Ormerod et al., 2010; Voeroesmarty et al., 2010). The direct measurement of stressors is often resource demanding, while point measurements are 
inadequate for assessing time-varying stressors. Therefore, river managers need indicators that 45 are easy to assess and help to identify and differentiate among various sources of impairment. Macroinvertebrates have been used for decades as ecological indicators for river water and habitat quality (Bonada et al., 2006; Charvet et al., 1998; Friberg et al., 2011; Kenney et al., 2009; Kolkwitz and Marsson, 1909; Rosenberg and Resh, 1993). The basic idea behind the use of the community composition of macroinvertebrates as bioindicators is that different taxa of 50 macroinvertebrates have different ecological requirements (niches) (Thompson and Townsend, 2006). Therefore, community assembly is influenced by environmental factors that act as socalled habitat filters (Poff, 1997). Hence, from knowledge about the habitat requirements of taxa we can infer the environmental conditions that lead to the observed community composition at a given site.

55 Classic examples are the Saprobic Index in Europe (DIN, 2004; Kolkwitz and Marsson, 1909; Rolauffs et al., 2004) and the Hilsenhoff Biotic Index in the USA (Hilsenhoff, 1987; Hilsenhoff, 1988) that were developed to indicate organic pollution. However, other widely applied macroinvertebrate indices, such as \%EPT (Weber, 1973) or diversity indices, indicate "general degradation" that might have several explanations (e.g. impaired hydromorphology, pollution 60 with organic matter or toxic substances, temperature stress) and are not necessarily suited to identifying site-specific causes of impairment (Murphy et al., 2013). The same applies to Reference Condition approaches, which were developed to assess the ecological state of an ecosystem (e.g. Stoddard et al., 2006).

The use of trait-based indices is a promising approach to help establish the causal relationships

65 between specific stressors and the macroinvertebrate community response (Doledec and Statzner, 2008; Menezes et al., 2010; Murphy et al., 2013; Statzner and Bêche, 2010), and to link structural and functional aspects of the ecosystem (Feio and Doledec, 2012; Haybach et al., 2004). An example of such a trait-based index already in use for river management is the

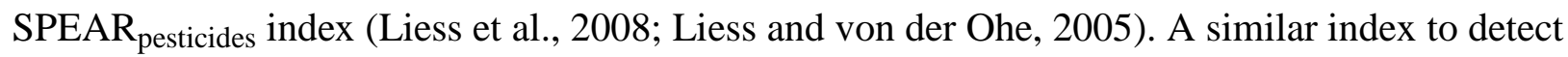
70 salinity stress in Australian rivers was developed by Schaefer et al. (2011). In recent years many ecologists have contributed to the collection of information about autecological traits of macroinvertebrates in databases (e.g. Poff et al., 2006; Schaefer et al., 2011; Schmidt-Kloiber and Hering, 2012; Statzner et al., 2007; Tachet et al., 2010). These databases include biological traits and life-history parameters (e.g. modes of locomotion, number 
75 of generations per year), but also ecological traits that describe habitat requirements of different taxa (e.g. temperature, salinity, current) based on experiments or on observations of species distributions . It seems straightforward to use this wealth of information about traits that are directly related to specific habitat characteristics for the development of new trait-based indices to detect stressors, e.g. following the SPEAR ("species at risk") concept (Liess and von der Ohe, 80 2005). In this regard, "stressors" are habitat characteristics that impose stress on the organisms and can be of anthropogenic or natural origin.

Here we use the term "trait-based index" for a function that combines abundance data of organisms with trait information about their sensitivity to a specific stressor. Ideally, trait-based indices are clearly linked to a single stressor and insensitive to other stressors. Under this 85 condition, it is possible to draw unequivocal conclusions about causes of impairment reflected in the taxonomic composition. However, this is only fulfilled if traits (or trait combinations as used

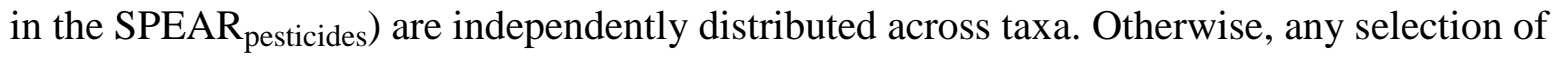
traits related to one stressor will cause a correlated trait-response for another stressor without any environmental influence. Therefore, we must carefully test against the general null hypothesis

90 that different traits are independently distributed across taxa. Neglecting possible inherent correlations may lead to false conclusions regarding factors affecting stream ecosystems. This problem will probably increase with a decreasing number of taxa that occur in a specific region simply because finding those taxa that help to differentiate between stressors in a smaller taxon pool is less likely. This inherent correlation will possibly also increase with decreasing

95 taxonomic resolution of the field data, as the autecological information is more specific on the species level than on an aggregated genus or family level. If trait correlations are indeed pronounced, they pose a serious issue for the use of trait-based indices in river management. Besides a potential inherent correlation among trait-based indices, environmental stressors also might be correlated. It is of major importance to differentiate these two sources of correlation to 100 infer the influence of different stressors correctly. While the response of a trait-based index to the target stressor is usually tested during the development of the index (e.g. Liess et al., 2008), and there are studies that test the overall correlation between different indices (e.g. Birk and Hering, 2006), we are not aware of studies that test the inherent correlation between different indices based on the species' sensitivities and distinguish it from the correlation of stressors. 
105 The aim of this paper is to analyse how stressor-specific trait-based indices are by analysing the degree of inherent correlation among different trait-based indices and its dependence on the taxon-pool. To this end, we test whether traits are independently distributed across taxa and analyse the correlation of indices from randomly generated communities. Furthermore, we show how to distinguish this inherent correlation among indices from a correlation between the corresponding environmental stressors. For this purpose, we not only analyse the taxon-pool of two catchments on the Swiss Plateau harbouring a number of different environmental stressors; we additionally examine a complete list of species occurring in Switzerland in the same Ecoregion as the two catchments as well. This helps us to evaluate the influence of the size of the taxon pool and the taxonomic resolution on inherent correlation. To illustrate this approach, we 115 analyse the SPEAR $\mathrm{R}_{\text {pesticides }}$ and the Saprobic Index that are established in river management. To increase the scope of the analyses to a wider range of natural and anthropogenic stressors, we constructed three trait-based indices for habitat tolerance of macroinvertebrates regarding temperature, flow velocity, and $\mathrm{pH}$ analogously to the SPEAR concept. We apply all indices to field samples of the two catchments mentioned above. With these analyses, we aim to stimulate 120 the development of new trait-based indices for river management and draw attention to possible pitfalls.

\section{Methods}

\subsection{Trait-based indices}

Trait data were derived from the database www.freshwaterecology.info, version 5.0, accessed on 125 2012-08-15 (Schmidt-Kloiber and Hering, 2012). We downloaded trait information on preferences regarding flow velocity (current), temperature, $\mathrm{pH}$, and saprobity. See appendix A1 for detailed information about the trait categories and their interpretation. The SPEAR pesticides $_{\text {s }}$ classification was downloaded via the R package "rspear", version 0.0.1 from E. Szoecs from "www.systemecology.eu/spear/spear-calculator/" on 2013-01-14.

We used the "species at risk" concept (Liess and von der Ohe, 2005) to calculate trait-based indices for water temperature, flow velocity, and $\mathrm{pH}$. Traits that are related to a specific stressor are combined and taxa are thereby classified as "sensitive" or "insensitive" with regard to the stressor. Based on this classification, the abundance of species at risk at a given site is calculated 
as the sum of the logarithm of the abundance of each species. The index is the ratio of the abundance of species at risk compared to the abundance of all species (eq.1).

$$
\text { Index }_{s}=\frac{\sum_{t}\left(\log \left(x_{t s}+1\right) \cdot y_{t}\right)}{\sum_{t} \log \left(x_{t s}+1\right)} \cdot 100
$$

with $x_{t s}$ abundance of taxon $t$ at site $s, y_{t}$ is 1 if taxon $t$ is classified as "sensitive", otherwise 0 . Increasing values of the index indicate a larger proportion of taxa that are sensitive to the stressor and therefore less influence of the stressor on community composition.

140 For environmental factors such as temperature or flow velocity, both high and low values can impose stress on different taxa. Taxa adapted to cold water may perceive high temperatures as "stress" and taxa adapted to warm water may perceive cold water as "stress". We can therefore define different indices for the same environmental factor.

In the trait database we used, several categories are distinguished for each trait. For example, the 145 temperature preference trait consists of five categories (very cold, cold, moderate, warm, or eurythermic). The traits are coded in terms of a ten-point assignment system, in the case of temperature, current and $\mathrm{pH}$ based on the known or estimated average distribution of each taxon (Euro-limpacs Consortium, 2009).

For each index, we defined the "stressor" (e.g. summer temperature $>18^{\circ}$ for index "WarmT") 150 and corresponding trait categories to distinguish between sensitive and insensitive taxa (Table 1). To convert the 10-point trait coding into the binary classification of sensitive and insensitive species, we first identified for each taxon the trait category with the highest number of points and assigned the taxon to this category. For example, we defined an index for warm water stress "WarmT" by classifying taxa that have the highest number of points assigned to the categories "very cold" or "cold" as sensitive and all other taxa as insensitive. We excluded taxa from the index that are not classified in the trait database (and if no trait information is available for any species of the same genus or family). The temperature and $\mathrm{pH}$ preferences were only available for EPT-taxa (Ephemeroptera, Plecoptera and Trichoptera) and chironomids (Chironomidae). In existing monitoring data, different taxa are typically identified at different taxonomic levels (e.g. species, genus, family, even order level). To assign traits to these different levels, we used the assumption of phylogenetic similarity. For levels higher than species, we used the average of 
available trait data from species belonging to the same higher taxonomic group. If trait information was missing for a certain taxon, we used averaged trait information of taxa from the same higher taxonomic level if possible.

165 Table 1: Trait-based indices for flow velocity, temperature, and $\mathrm{pH}^{*}$

\begin{tabular}{llll}
\hline index & stressor & $\begin{array}{l}\text { traits of insensitive } \\
\text { taxa }(\mathbf{y}=\mathbf{0})\end{array}$ & $\begin{array}{l}\text { traits of sensitive } \\
\text { taxa }(\mathbf{y}=\mathbf{1})\end{array}$ \\
\hline LowFlow & standing to slow flowing water & lib, lip, lrp, rlp, ind & rhp, rhb \\
FastFlow & fast flowing water & rhp, rhb, ind & lrp, rlp, lib, lip \\
ColdT & cold water $<10^{\circ} \mathrm{C}$ & vco, cold, eut & mod, warm \\
WarmT & warm water $>18^{\circ} \mathrm{C}$ & warm, eut & vco, cold, mod \\
LowpH & acidic water $\mathrm{pH}<7$ & aci, ind & neu \\
HighpH & neutral to alkaline water $\mathrm{pH} \geq 7$ & neu, ind & aci \\
\hline
\end{tabular}

*lib=limnobiont, lip=limnophil, lrp= limno to rheophil, rlp= rheo to limnophil, rhp= rheophil, $\mathrm{rhb}=$ rheobiont, ind $=$ indifferent, $\mathrm{vco}=$ very cold, $\bmod =$ moderate, eut=eurythermic, neu=neutral to alkaline, aci= acid

In both cases we used at least family level taxonomic data. Taxa that were identified at higher 170 levels or taxa for which trait information from taxa of the same family was not available were excluded from the calculation. This simple assumption certainly decreases the accuracy of the trait information and must be reviewed by taxonomic specialists before using the indices in

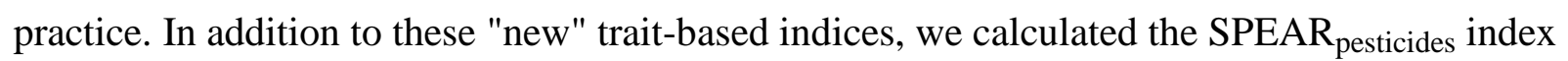
(Liess and von der Ohe, 2005) and the Saprobic Index (DIN, 2004; Kolkwitz and Marsson, 1909;

175 Rolauffs et al., 2004) by using the Austrian classification of taxa (SI_AT) (Moog, 2002).

\subsection{Macroinvertebrate monitoring data}

We applied the trait-based indices to macroinvertebrate data collected in two catchments of the Swiss Plateau (Mönchaltorfer Aa and Gürbe). Sampling details are given in Robinson et al. (2014). Samples were collected in October 2010, April 2011, and August 2011 at 11 sites in each catchment using a Hess sampler $\left(0.048 \mathrm{~m}^{2}, 250 \mu \mathrm{m}\right.$ mesh $)$ and identified to the lowest practical taxonomical level (8 taxa on species level, 21 on genus level, 24 on family level, see Appendix A2). In the following, we use data averaged over the three sampling dates. However, we tested if results are different when using only the samples from April 2011 since SPEAR pesticides $_{\text {is }}$ 
usually based on spring samples. Additional water quality and habitat parameters (e.g. total organic carbon (TOC), carbonate alkalinity, conductivity, $\mathrm{NH}_{4}-\mathrm{N}, \mathrm{NO}_{3}-\mathrm{N}$, dissolved-N, particulate-N, $\mathrm{PO}_{4}-\mathrm{P}$, dissolved-P, and particulate-P, water temperature, flow velocity) were measured at the same time (grab samples) (Robinson et al., 2014).

\subsection{Comparison of indices with environmental gradients}

Before analysing the correlation structure between indices, we compared the indices calculated 190 from field samples to field data of the environmental conditions that corresponded most closely to the ones that were used for the trait classification (visually and with correlation analysis). This is a plausibility check and cannot substitute for more in-depth testing of the sensitivity of these indices regarding the corresponding stressor, which is beyond the scope of this study. This task would require measurements of exactly those environmental variables that were used to define 195 the trait categories in the database and the coverage of a wide range of the environmental gradients.

\subsection{Analysis of correlation among indices}

First, we calculated trait-based indices for the 22 field samples and assessed the correlation between trait-based indices using Pearson's correlation coefficient. As mentioned above, this correlation between indices can be inherent because taxa can be sensitive to multiple stressors, or it can originate from correlation between the stressors themselves. To differentiate between these possible sources, we performed the following three steps.

Step 1: To assess the potential for inherent correlation of different trait-based indices, we analysed the classification of taxa as "sensitive" or "insensitive" as defined in the different indices. If traits that are used for two different indices (corresponding to two different stressors) are not independently distributed across taxa, indicating that taxa are sensitive (or insensitive) to both stressors, this will lead to an inherent correlation between the two indices and therefore to a response of one index to the stressor of the other index. To test if this inherent correlation of indices depends on the size of the taxon pool and its taxonomic resolution, we used two different taxon pools, which vary substantially in size and resolution.

a) The taxon pool "Obs_GM" consists of all 53 taxa (species, genus or family level) that were observed in at least one of the field samples from the two catchments (Gürbe, Mönchaltorfer Aa) (Robinson et al., 2014). The taxa list is given in Appendix A2. 
b) The taxon pool "CH_ER8" consists of all species of the European ecoregion 8 (Western 215 Highlands) (http://www.eea.europa.eu/data-and-maps/figures/ecoregions-for-rivers-and-lakes) that occur in Switzerland, downloaded from www.freshwaterecology.info (558 species). For each taxon pool, we analysed contingency tables representing the frequencies of taxa that are sensitive to both stressors, insensitive to both stressors, or sensitive to only one of the stressors for pairwise combinations of indices. We tested the null hypothesis that the classification of taxa in different indices is independent by applying a two-sided Fisher's Exact Test for count data (Fisher, 1922.). Since the question concerning in which cases p-values should be corrected to control the type I error for multiple tests remains under debate, we calculated p-values with and without Holm's correction (Cabin and Mitchell, 2000; Holm, 1979; Wright, 1992).

Step 2: To directly estimate the inherent correlation between indices due to correlation in the classification of taxa, we analysed the correlation of indices from random samples. As in Step 1, we used both taxon pools to analyse if this inherent correlation depends on the size of the taxon pool and its taxonomic resolution. We performed Monte Carlo simulations to generate random samples representing hypothetical field samples $(n=1000$, see appendix A3 for a detailed description of the sampling procedure). We calculated the trait-based indices for these samples, Pearson's correlation coefficient, and p-values for pairs of trait-based indices with and without Holm's correction.

Step 3: We finally assessed the probability for the null-hypothesis that the observed correlation of indices in field samples resulted only from inherent correlation and not from a correlation of stressors using Monte Carlo simulations. For each pair of indices, we compared the single correlation coefficient of the index-pair in the 22 field samples ("Obs_GM") to the distribution of correlation coefficients resulting from 1000 sets of 22 random samples from the same taxon pool "Obs_GM". The random taxon samples were derived in the same way as in step 2. For each of the 1000 replicates, the index values were calculated for each of the 22 samples as well as Pearson's correlation coefficient for each index-pair. The 22 field samples differ with respect to 240 the stressor conditions and hence, the single correlation coefficient for each index-pair includes both, inherent correlation and potential correlation of stressors. In contrast, correlation in the 1000 sets of 22 random samples includes only inherent correlation. If the correlation of stressors contributes substantially to the correlation of the indices from field-samples, we would expect that the observed correlation is larger than in the random samples. Therefore, we calculated the 
proportion of correlation coefficients from the random samples that were larger (or smaller in case of a negative correlation) than the correlation coefficient from field samples. From this we get the probability for the null hypothesis that the correlation between trait-based indices in the field samples is only due to the inherent correlation of indices (one-sided test). All calculations were done with the statistics and graphics software R (R Development Core Team, 2011) using 250 the packages "sampling", and "Hmisc".

\section{Results}

\subsection{Plausibility of the trait-based indices suggested}

The correlation of indices of the 22 field sites with those measured environmental variables that corresponded most closely to the ones that were used for the trait classification either had the expected sign (negative or positive) or they were weak (Fig. A-1). There was no correlation between the flow indices (LowFlow, FastFlow) and flow velocity measured at base flow. There was a negative correlation of -0.61 for the warm-water stress index (WarmT) with mean water temperature and no correlation between the cold-water stress index (ColdT) with mean water temperature. The $\mathrm{pH}$ indices correlated with measured alkalinity $(\mathrm{r}=-0.53$ for $\mathrm{LowpH}$ and $\mathrm{r}=0.27$

260 for HighpH, despite the fact that all $\mathrm{pH}$ values were above 7) and the correlation showed the expected sign. The Saprobic Index (SI_AT) correlated with the expected sign ( $\mathrm{r}=0.48)$ with total organic carbon (TOC), as well as with several nitrogen and phosphorus species and total and dissolved organic matter (not shown).

\subsection{Correlation analysis}

265 Data from the field study revealed some strong correlations between trait-based indices for different stressors (Fig. 1a, Table 2a, Table A-2, Fig. A-2). Strong correlations were especially observed between SPEAR $_{\text {pesticides }}$ and the warm-water stress index WarmT ( $\left.r=0.9\right)$, and between SPEAR $_{\text {pesticides }}$ and the Saprobic Index ( $\left.\mathrm{r}=-0.9\right)$. Only the cold-water stress index (ColdT) and the index for neutral to high $\mathrm{pH}$-conditions $(\mathrm{HighpH})$ had an $r<|0.30|$ with $\mathrm{SPEAR}_{\text {pesticides. Only }}$ 270 the low-flow stress index (LowFlow) and the cold-water stress index (ColdT) had an $r<|0.30|$ with the Saprobic Index. Results of using pooled samples from different dates or only spring samples were similar (Figs. A-2 and A-3). In the next steps we analysed the sources of the observed correlation. 
4275 a) field samples
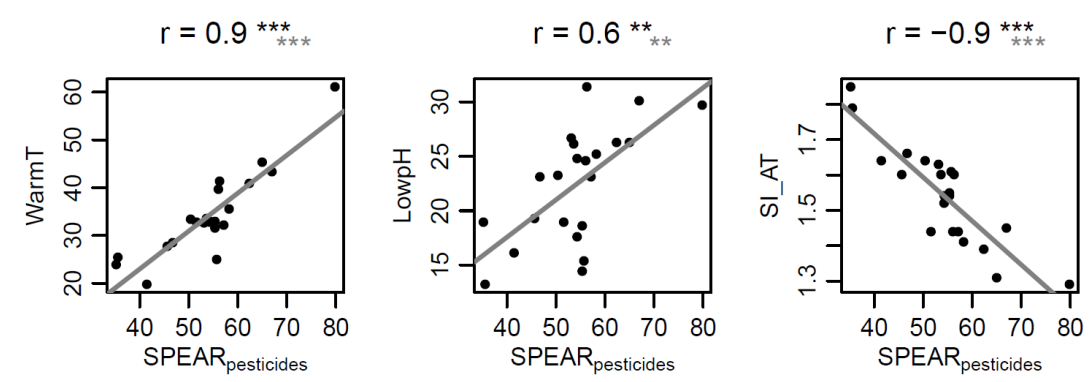

b) "Obs_GM" taxon pool, random sampling, $n=1000$
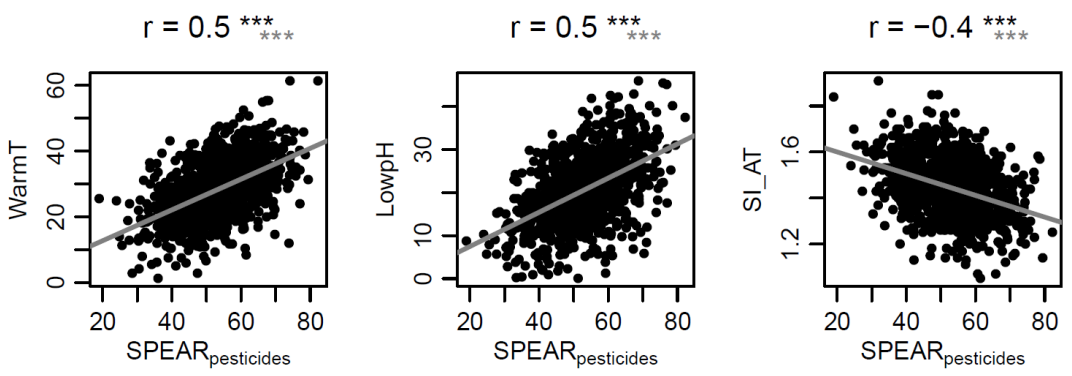

c) "CH_ER8" taxon pool, random sampling, $\mathrm{n}=1000$
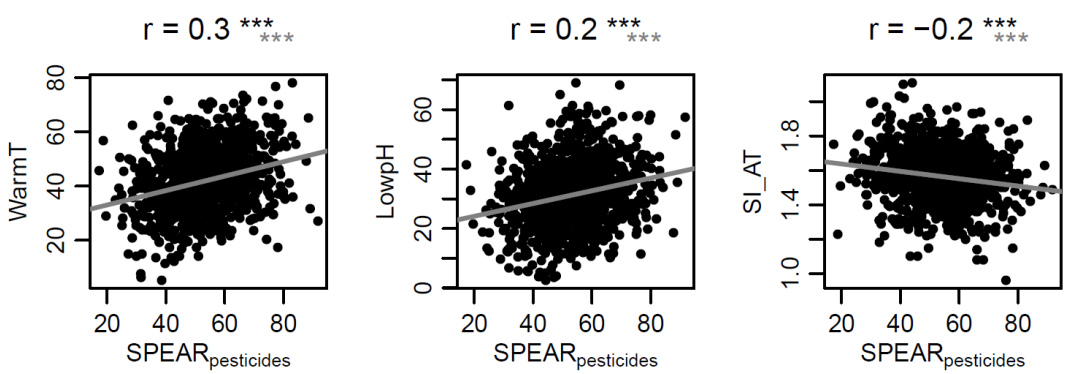

Fig. 1: Correlation of the indices WarmT, LowpH, and the Saprobic Index (SI_AT) with SPEAR $_{\text {pesticides }}$ for (a) the field samples, (b) random samples from the same taxon pool ("Obs_GM"), and (c) random samples from the "CH_ER8"-taxon pool. Pearson's correlation coefficient $r$ : stars indicate significance of correlation, black without and grey with Holms correction, regression line in grey. 
Table 2: Pearson's correlation coefficients and uncorrected p-values for the correlation between SPEAR $_{\text {pesticides, }}$ the Saprobic Index SI_AT and other trait-based indices (Table 1); r values $>|0.3|$ are highlighted in grey.

\begin{tabular}{lcccccc}
\hline & $\begin{array}{l}\text { a) field samples } \\
(\mathrm{n}=22)\end{array}$ & \multicolumn{3}{c}{$\begin{array}{l}\text { b) "Obs_GM" } \\
\text { taxon pool, } \\
\text { random sampling } \\
(\mathrm{n}=1000)\end{array}$} & $\begin{array}{l}\text { c) "CH_ER8" } \\
\text { taxon pool, } \\
\text { random sampling } \\
(\mathrm{n}=1000)\end{array}$ \\
\hline & $\mathrm{r}$ & $\mathrm{p}$ & $\mathrm{r}$ & $\mathrm{p}$ & $\mathrm{r}$ & $\mathrm{p}$ \\
\hline Spear vs. LowFlow & 0.38 & 0.09 & -0.002 & 0.96 & 0.19 & $<0.001$ \\
Spear vs. FastFlow & -0.56 & 0.01 & 0.09 & 0.006 & 0.33 & $<0.001$ \\
Spear vs. ColdT & 0.28 & 0.21 & 0.22 & $<0.001$ & -0.29 & $<0.001$ \\
Spear vs. WarmT & 0.89 & $<0.001$ & 0.53 & $<0.001$ & 0.28 & $<0.001$ \\
Spear vs. LowpH & 0.65 & 0.001 & 0.50 & $<0.001$ & 0.23 & $<0.001$ \\
Spear vs. HighpH & -0.19 & 0.39 & 0.22 & $<0.001$ & 0.20 & $<0.001$ \\
Spear vs. SI_AT & -0.88 & $<0.001$ & -0.37 & $<0.001$ & -0.16 & $<0.001$ \\
SI_AT vs. LowFlow & -0.18 & 0.41 & -0.29 & $<0.001$ & -0.28 & $<0.001$ \\
SI_AT vs. FastFlow & 0.41 & 0.06 & -0.11 & $<0.001$ & 0.12 & $<0.001$ \\
SI_AT vs. ColdT & -0.18 & 0.43 & -0.01 & 0.68 & 0.34 & $<0.001$ \\
SI_AT vs. WarmT & -0.77 & $<0.001$ & -0.27 & $<0.001$ & -0.14 & $<0.001$ \\
SI_AT vs. LowpH & -0.49 & 0.02 & -0.15 & $<0.001$ & 0.01 & 0.86 \\
SI_AT vs. HighpH & 0.41 & 0.06 & -0.01 & 0.81 & -0.02 & 0.63 \\
\hline
\end{tabular}

290 Step 1: In summary, this first step demonstrated that in most cases the trait combinations were not independently distributed across taxa (Fig. 2). This strongly depended on the size and taxonomic resolution of the taxon pool. Accordingly, one cannot consider the different indices a priori as (statistically) independent.

We exemplarily showed the contingency tables for the classification of taxa for the combination 295 of $\mathrm{SPEAR}_{\text {pesticides }}$ with the other indices for both taxon pools (Fig. 2). For comparison, we also showed contingency tables for the combination of indices that belong to the same trait (e.g. WarmT and ColdT) because we would expect those not to be independent. 
a) "Obs_GM" taxon pool
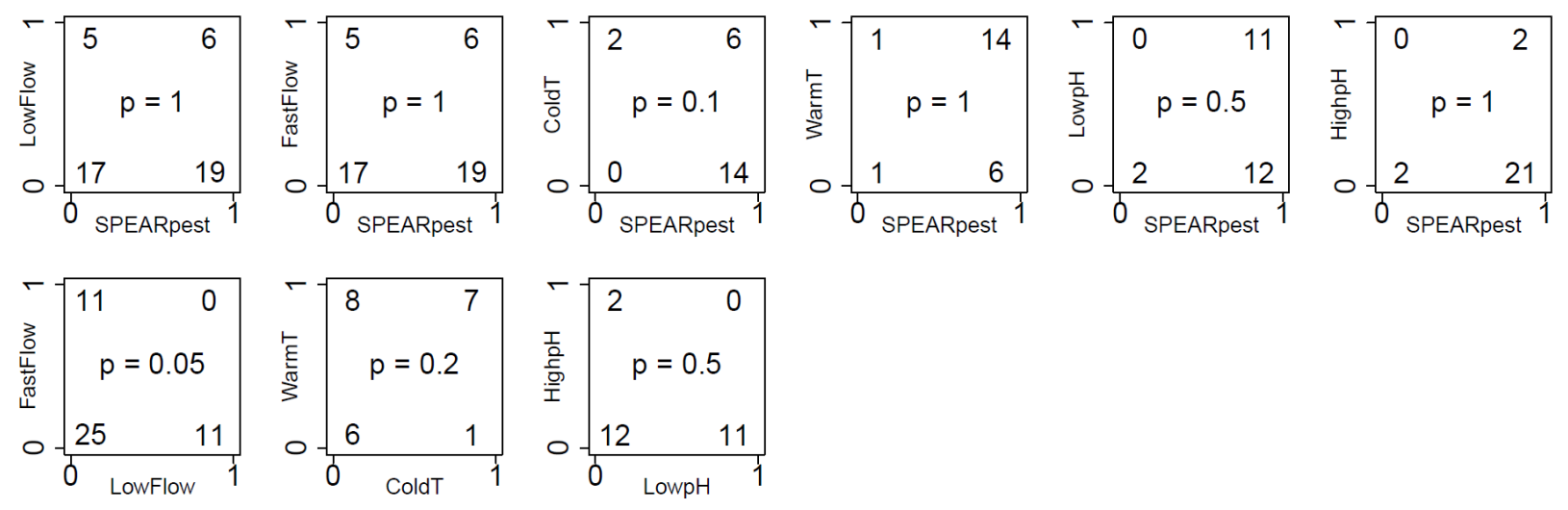

b) "CH_ER8" taxon pool
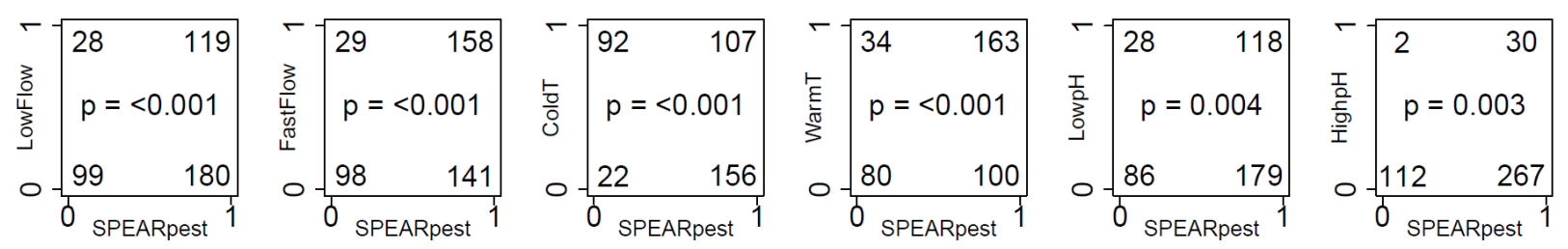

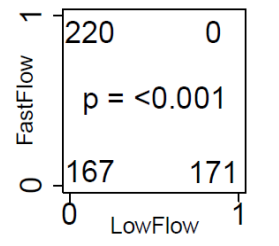

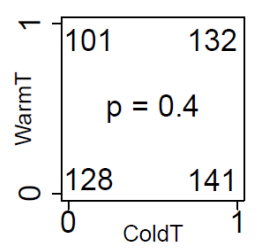

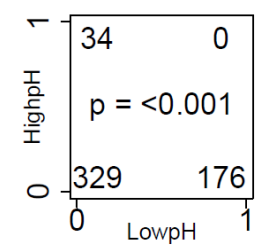

Fig. 2: Contingency tables for the classification of taxa from (a) "Obs_GM" taxon pool and (b) "CH_ER8" taxon pool. Trait-based indices are defined in Table 1; 0="insensitive" and 1="sensitive"; two-sided Fisher's Exact Test for the null hypothesis that the classification of taxa in different indices is independent; $p$-values $<0.05$ indicate that the null hypothesis is not supported.

Within the "Obs_GM" taxon pool, in several cases at least one of the four categories was represented by a single taxon only or was missing (Fig. 2a). Nevertheless, Fisher's Exact Test lead to $\mathrm{p}$-values $>0.05$, thus there was not sufficient evidence to reject the null hypothesis of independence. However, due to the small sample size (between 22 and 47 classified taxa) the test is not particularly strong. An exception is the combination of both flow indices with $\mathrm{p}<0.05$, which justifies a rejection of the null hypothesis of independence. 
Within the "CH_ER8" taxon pool, in most cases several species were represented in each

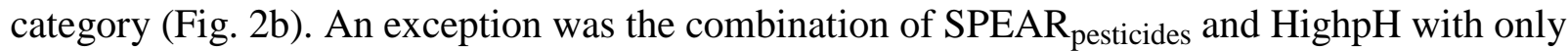
two taxa in the category "01" (i.e. classified as insensitive in SPEAR pesticides and sensitive in HighpH). This is partly because trait information for $\mathrm{pH}$ indices was not available for all taxa. The total number of classified taxa ranged between 377 and 558. For the combination of both flow indices and both $\mathrm{pH}$ indices, respectively, no taxa were classified as sensitive in both indices.

Fisher's Exact Test lead to p-values < 0.05 (with and without Holm's correction) for all pairs of

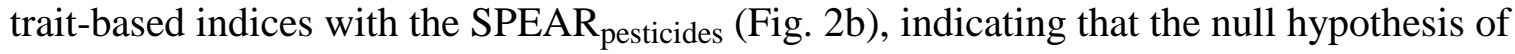
independence should be rejected. This was also the case for the combination of both flow- and both $\mathrm{pH}$-indices, where the classification of both indices is based on the same trait. Interestingly, this was not the case for the combination of warm- and cold-temperature stress indices, despite the fact that they were based on the same trait. In contrast to the pairs of flow- and $\mathrm{pH}$-indices, there are taxa that were classified as sensitive in both indices, namely the taxa that prefer moderate water temperatures.

Step 2: The inherent correlation between indices from random samples (excluding effects of stressor correlation) depends on the taxon pool (Table 2bc, Figs. 1bc, A-4 and A-5). For the "Obs_GM" taxon pool, the highest correlation of SPEAR pesticides was found with the index for warm water stress (WarmT) with $r=0.53$, with the lowpH index with $r=0.5$ and with the Saprobic Index with $\mathrm{r}=-0.37$. All other correlations coefficients were below $|0.3|$ (Table $2 \mathrm{~b}$ and Fig. A-4).

For the "CH_ER8" taxon pool, the highest (absolute) correlation was found between the 335 Saprobic Index and the temperature index for cold water stress (ColdT) with $r=0.34$ and

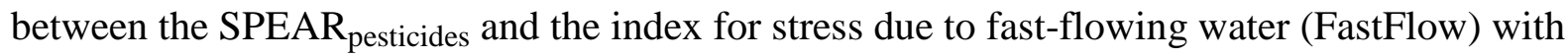
$r=0.33$. All other correlation coefficients were below $|0.3|$. Due to the large sample size, all correlations were significant $(p<0.05)$, with the exception of the correlation between the Saprobic Index and the pH-indices, with r-values below $|0.05|$ and p-values $>0.5$.

Although the "Obs_GM" taxon pool is much smaller and has a lower taxonomic resolution, 8 out of 13 correlations had the same sign as observed in the "CH_ER8" taxon pool. In both taxon pools there were inherent correlation structures between trait-based indices that are not based on 
the same trait. However, although most of them were statistically highly significant, they explained only a limited part of the variability indicated by $\mathrm{r}^{2}$ values below 0.3 (corresponding to $\mathrm{r}$ values below $|0.55|$, Tab. 2B and C).

Step 3: A comparison of field and random samples indicated the importance of the correlation of stressors. The correlation of SPEAR pesticides $_{\text {with other indices was stronger in the field samples }}$ $\left(r_{\text {field }}\right)$ than the mean of the random samples $\left(\bar{r}_{\text {samp }}\right)$ (Fig. 3). This holds especially for the indices FastFlow, WarmT, and the Saprobic Index. The probability that the observed correlation in field samples is only due to inherent correlation was below 0.05 for all cases except for the

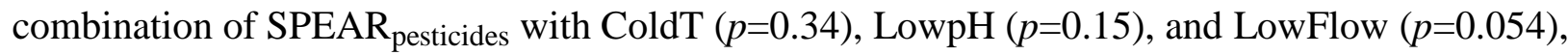
respectively. These results indicate a substantial contribution of the correlation of stressors in the field samples to the overall correlation. This was confirmed by correlating environmental conditions directly (Robinson et al., 2014).
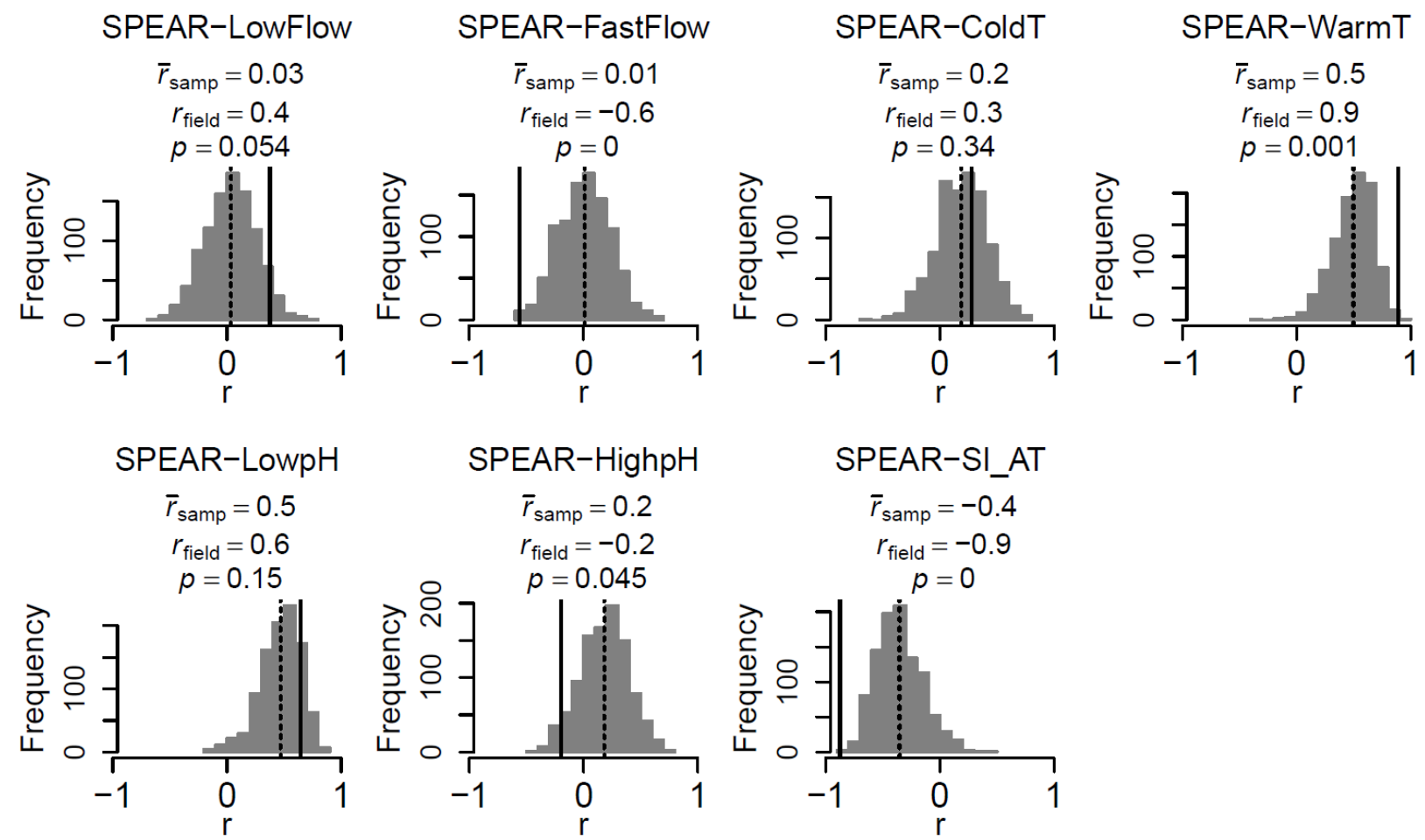

Fig.3: Histogram of Pearson's correlation coefficients $r_{\text {rsamp }}$ of several trait-based indices with the

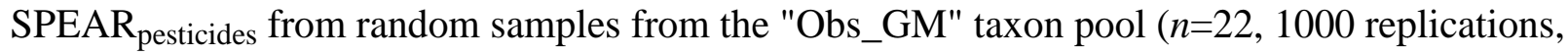
grey area); solid vertical line: correlation coefficient of field samples $r_{\text {field }}(n=22)$; dashed line: mean $r_{\text {rsamp; }} ; p$ gives the probability that the observed correlation is only due to the inherent correlation in the classification of taxa. 


\section{Discussion}

Trait-based indices have received much attention in research and practice over the last few years as a means to provide valuable insights into stressors affecting aquatic ecosystems. Such indices are suited as an inexpensive and rapid assessment tool if biological monitoring data exists. An example is the SPEAR pesticides (Liess and von der Ohe, 2005), which is already used by legal authorities in Switzerland. Despite its imperfection, we believe it is now time to make use of the information on ecological traits regarding habitat requirements that is currently made publicly available (e.g. Schmidt-Kloiber and Hering, 2012). The development of trait-based indices for habitat requirements such as temperature, current and $\mathrm{pH}$ seems straightforward. To evaluate their sensitivity and precision, more comprehensive testing would be required. This includes a) a better estimation of traits at genus and family level since the taxonomic resolution of monitoring data rarely goes to the species level for all taxonomic groups, b) a better match between environmental conditions measured in the field and environmental conditions used to define 375 traits and c) a large dataset which covers wide environmental gradients.

The value of such indices for habitat requirements is not only to detect stress induced by the related environmental gradients of natural or anthropogenic origin. They can also help us to understand the influence of various environmental stressors on the established trait-based indices, which can act as confounding factors, as demonstrated in this study. Obviously, the precision of trait-based indices depends on the precision of the trait data that are used to construct the indices (which are often derived from species-distribution data and not from experimental studies) and on the quality of available field monitoring data (e.g. representativeness in time and space, taxonomic resolution). In this study, we emphasize that the proper use of trait-based indices also relies on a sound understanding of how they depend on the 385 presence of other stressors. For example, SPEAR ${ }_{\text {pesticides }}$ can be lower at sites with strong hydromorphological degradation regardless of a potential source of pesticide contamination (Bunzel et al., 2013; Bunzel et al., 2014). We have shown here that such dependencies exist in a statistical sense and that such relationships are probably more the rule than the exception. This phenomenon can be partially explained by the physiology of the organisms. For example, 390 organisms that require cold water are often also bound to zones with high current velocity and a 
good water quality regarding organic matter since they need high oxygen concentrations in the water due to their respiratory system. The occurrence of so-called trait syndromes (combination of traits that are characteristic of a single environmental condition) is discussed e.g. in Poff et al. (2006) and Statzner and Bêche (2010). However, including only those taxa that are sensitive to only one stressor is unrealistic due to the limited number of taxa in each sample. The inherent correlation between trait-based indices was more severe for the smaller taxon pool with a lower taxonomic resolution. This is plausible since sensitive, stressor specific indicator taxa are more likely to be found in larger taxon pools with a higher resolution (species level). In areas with a long history of different human impacts, such as the Swiss Plateau, we can expect a loss of 400 regional biodiversity and therefore a loss of indicator taxa for specific stressors.

Our results have shown that trait-based indices calculated from field samples may strongly correlate, even if they are intended to indicate different stressors. This correlation can be partly attributed to inherent correlation in the classification of taxa (which depends on the regional taxon pool and the taxonomic resolution) and partly to a correlation of the stressors in the environment themselves. For example, the correlation coefficient between the SPEAR ${ }_{\text {pesticides }}$ and the Saprobic Index (SI_AT) in our field samples is -0.88 , the mean correlation coefficient from random samples of the same taxon pool is -0.37 , and the probability that the observed correlation in the field samples is only due to correlation in the classification of taxa is $<0.001$. These results indicate that in the study area there is indeed a strong correlation between the two stressors 410 (pesticides and organic pollution). Therefore, both would have to be addressed to reduce anthropogenic effects on the community composition.

\section{Conclusions}

The correlation between trait-based indices for different stressors as described in this paper has several implications. First, the indication of several stressors at a site does not necessarily imply that all the stressors actually affect the ecosystem. Such a conclusion may only be drawn if one tests the correlation of trait-based indices against an appropriate null hypothesis, as we have done in this study. We suggest an empirical distribution obtained from random sampling of the entire taxon pool from the study area, which should also cover sites unaffected by the stressors in question, as an appropriate solution.

420 Second, one must be aware of such dependencies also in cases where one only focuses on one single index and uses that for learning about the possible influence of the corresponding 
environmental stressor. Due to the possible statistical dependencies between trait-based indices, a spatial pattern in one index may actually be caused by the (hidden) effect of another stressor. Our findings illustrate that results from trait-based indices should be interpreted with caution, 425 especially in regions with low biodiversity where only a reduced taxon pool is able to colonize available habitats. On the other hand, as we have shown in step 3 of our analysis, part of this correlation can indeed be attributed to a correlation of stressors that can be observed in many catchments on the Swiss Plateau and in Central Europe in general which are heavily influenced by human activity. Often, small headwater streams exhibit lower summer temperatures, higher current velocities, and lower loadings of organic matter, nutrients and pesticides than downstream parts of the river basins.

Third, during the development of trait-based indices it is very important to test for confounding factors and - if they cannot be completely avoided - to communicate them to potential end-users. In this paper we draw attention to sources of potential correlation between trait-based indices and 435 provide a strategy to deal with this challenge to avoid misinterpretation.

\section{Acknowledgements:}

The authors acknowledge Julia Stefanovic for help in data processing and Carlo Albert for inspiring discussions on methodological issues. We graciously thank Christopher Robinson, Simone Baumgartner, Christa Jolidon, Alfred Lück, Michael Scheurer and Nico Ghielmetti for field and laboratory processing of the field samples. We thank the Aua lab of Eawag for completing the water chemistry analyses. We thank Christopher Robinson and Cédric Mondy for helpful comments on an earlier version of the manuscript. Four anonymous reviewers are acknowledged for constructive comments, which helped us improving the manuscript. This study is part of the project "iWaQa: Integrated River Water Quality Management" funded by the Swiss

445 National Science Foundation (NRP61 on Sustainable Water Management) (no. 406140_125866/1).

\section{References:}

Birk S, Hering D. Direct comparison of assessment methods using benthic macroinvertebrates: a contribution to the EU Water Framework Directive intercalibration exercise. $450 \quad$ Hydrobiologia 2006; 566: 401-415.

Bonada N, Prat N, Resh VH, Statzner B. Developments in aquatic insect biomonitoring: A comparative analysis of recent approaches. Annual Review of Entomology. 51, 2006, pp. 495-523. 
Bunzel K, Kattwinkel M, Liess M. Effects of organic pollutants from wastewater treatment plants on aquatic invertebrate communities. Water Research 2013; 47: 597-606.

Bunzel K, Liess M, Kattwinkel M. Landscape parameters driving aquatic pesticide exposure and effects. Environmental Pollution 2014; 186: 90-97.

Cabin RJ, Mitchell RJ. To Bonferroni or Not to Bonferroni: When and How Are the Questions. Bulletin of the Ecological Society of America 2000; 81: 246-248.

Charvet S, Kosmala A, Statzner B. Biomonitoring through biological traits of benthic macroinvertebrates: perspectives for a general tool in stream management. Archiv Fuer Hydrobiologie 1998; 142: 415-432.

DIN. 38410-1:2004-10, German standard methods for the examination of water, waste water and sludge - Biological-ecological analysis of water (group M) - Part 1: Determination of the saprobic index in running waters (M 1). 2004.

Doledec S, Statzner B. Invertebrate traits for the biomonitoring of large European rivers: an assessment of specific types of human impact. Freshwater Biology 2008; 53: 617-634.

Euro-limpacs Consortium. Taxa and Autecology Database for Freshwater Organisms. Eurolimpacs project (contract no. GOCE-CT-2003-505540), Workpackage 7 - Indicators of ecosystem health, Task 4, www.freshwaterecology.info [version 4.0, 12.2009]). 2009.

Feio MJ, Doledec S. Integration of invertebrate traits into predictive models for indirect assessment of stream functional integrity: A case study in Portugal. Ecological Indicators 2012; 15: 236-247.

Fisher RA. On the interpretation of $\chi 2$ from contingency tables, and the calculation of p. Journal of the Royal Statistical Society Series a-Statistics in Society 1922.; 85 87-94.

Friberg N, Bonada N, Bradley DC, Dunbar MJ, Edwards FK, Grey J, et al. Biomonitoring of Human Impacts in Freshwater Ecosystems: The Good, the Bad and the Ugly. In: Woodward G, editor. Advances in Ecological Research, Vol 44. 44, 2011, pp. 1-68.

Haybach A, Schöll F, König B, Kohmann F. Use of biological traits for interpreting functional relationships in large rivers. Limnologica - Ecology and Management of Inland Waters 2004; 34: 451-459.

Hilsenhoff WL. An improved biotic index of organic stream pollution. Great Lakes Entomologist 1987; 20: 31-39.

Hilsenhoff WL. Seasonal Correction Factors for the Biotic Index. Great Lakes Entomologist 1988; 21: 9-13.

Holm S. A simple sequentially rejective multiple test procedure. Scandinavian Journal of Statistics 1979; 6: 65-70.

Kenney MA, Sutton-Grier AE, Smith RF, Gresens SE. Benthic macroinvertebrates as indicators of water quality: The intersection of science and policy. Terrestrial Arthropod Reviews 2009; 2: 99-128.

Kolkwitz R, Marsson M. Ökologie der tierischen Saprobien. Beiträge zur Lehre von der biologischen Gewässerbeurteilung. Internationale Revue der gesamten Hydrobiologie und Hydrographie 1909; 2: 126-152.

Liess M, Schaefer RB, Schriever CA. The footprint of pesticide stress in communities-Species traits reveal community effects of toxicants. Science of the Total Environment 2008; 406: 484-490. 
Liess M, von der Ohe PC. Analyzing effects of pesticides on invertebrate communities in streams. Environmental Toxicology and Chemistry 2005; 24: 954-965.

Menezes S, Baird DJ, Soares AMVM. Beyond taxonomy: a review of macroinvertebrate traitbased community descriptors as tools for freshwater biomonitoring. Journal of Applied Ecology 2010; 47: 711-719.

Moog O. Fauna Aquatica Austriaca, Lieferungen 1995, 2002. Wasserwirtschaftskataster, Bundesministerium fuer Land- und Forstwirtschaft, Umwelt und Wasserwirtschaft, Wien., 2002.

Murphy JF, Davy-Bowker J, McFarland B, Ormerod SJ. A diagnostic biotic index for assessing acidity in sensitive streams in Britain. Ecological Indicators 2013; 24: 562-572.

Ormerod SJ, Dobson M, Hildrew AG, Townsend CR. Multiple stressors in freshwater ecosystems. Freshwater Biology 2010; 55: 1-4.

Poff NL. Landscape Filters and Species Traits: Towards Mechanistic Understanding and Prediction in Stream Ecology. Journal of the North American Benthological Society 1997; 16: 391-409.

Poff NL, Olden JD, Vieira NKM, Finn DS, Simmons MP, Kondratieff BC. Functional trait niches of North American lotic insects: traits-based ecological applications in light of phylogenetic relationships. Journal of the North American Benthological Society 2006; 25: 730-755.

R Development Core Team. R: A language and environment for statistical computing. R Foundation for Statistical Computing, Vienna, Austria, 2011.

Robinson CT, Schuwirth N, Baumgartner S, Stamm C. Spatial relationships between land-use, habitat, water quality and lotic macroinvertebrates in two Swiss catchments. Aquatic Sciences 2014; 76: 375-392.

Rolauffs P, Stubauer I, Zahradkova S, Brabec K, Moog O. Integration of the saprobic system into the European Union Water Framework Directive - Case studies in Austria, Germany and Czech Republic. Hydrobiologia 2004; 516: 285-298.

Rosenberg DM, Resh VH. Freshwater Biomonitoring and Benthic Macroinvertebrates. New York, U.S.A: Chapman and Hall, 1993.

Schaefer RB, Kefford B, Metzeling L, Liess M, Burgert S, Marchant R, et al. A trait database of stream invertebrates for the ecological risk assessment of single and combined effects of salinity and pesticides in South-East Australia. Science of The Total Environment 2011; 409: 2055-2063.

530 Schmidt-Kloiber A, Hering D. www.freshwaterecology.info - the taxa and autecology database for freshwater organisms, version 5.0., 2012.

Statzner B, Bêche LA. Can biological invertebrate traits resolve effects of multiple stressors on running water ecosystems? Freshwater Biology 2010; 55: 80-119.

Statzner B, Bonada N, Dolédec S. Conservation of taxonomic and biological trait diversity of European stream macroinvertebrate communities: a case for a collective public database. Biodiversity and Conservation 2007; 16: 3609-3632.

Stoddard JL, Larsen DP, Hawkins CP, Johnson RK, Norris RH. Setting expectations for the ecological condition of streams: the concept of reference condition. Ecological Applications 2006; 16: 1267-1276. 
4540 Tachet H, Richoux P, Bournaud M, Usseglio-Polatera P. Invertébrés d'eau douce systématique, biologie, écologie. Paris: CNRS Editions, 2010.

Thompson R, Townsend C. A Truce with Neutral Theory: Local Deterministic Factors, Species Traits and Dispersal Limitation Together Determine Patterns of Diversity in Stream Invertebrates. Journal of Animal Ecology 2006; 75: 476-484. 